\title{
ON THE EVOLUTION OF QUASARS AND THEIR REMNANTS
}

\author{
P. KAFKA \\ Max-Planck-Institut für Physik und Astrophysik, Munich, Germany
}

\begin{abstract}
The evolution of massive cores in the post-quasar phase is considered.
\end{abstract}
Obviously galaxies are able to produce nuclear condensations of about $10^{8}$ (or more) solar masses within a light-month (or less). If we disregard the possibility that such nuclei are 'retarded cores' in universal expansion, or places where "new matter is poured into our world", we are faced with the problem, how gravitation manages to defeat rotation. It is clear, that angular momentum must be transported from inner to outer regions, such that the former can shrink, while the latter expand. Hence, we have to start with more total mass than we finally need.

If the original accumulation of mass is already fragmented into stars, the desired transport must be undertaken by their gravitational interaction. The stellar-dynamical 'evaporation' of stars from a cluster makes it shrink approximately along lines $R M^{-2}=$ const in the mass-radius diagram. Thus the process might produce quasarlike nuclei in galactic centers. It was proposed and discussed by various authors. (e.g. Gold, 1965; Spitzer and Saslaw, 1966; von Hoerner, 1968). The shrinking time predicted by classical stellar dynamics is far too long and only at the desired final state does it become as low as the age of the universe. However, since the influence of a broad mass function and the effect of binary formation have been discussed as possibilities to lower the time scale, it seems that the stellar dynamical process may not yet be excluded.

If the original large mass is not fragmented into stars, or if a dense interstellar material is left (or newly produced), friction and turbulence can transport angular momentum outward. More efficient, however, will be the winding up of magnetic fields in a rotating disk of gas. This mechanism was discussed by various authors (cf. Kardashev, 1964; Piddington, 1964, 1967; Ginzburg and Ozernoy, 1964; Pikelner, 1965; Ozernoy, 1966, and contribution to this symposium). More detailed examination is in progress, also on a stellar scale, where the mechanism will be important for supernova explosions.

Now let us assume that one of the mechanisms produced a condensation of several $10^{8}$ solar masses within only $\frac{1}{10} \mathrm{pc}$. The question is then, how this object liberates energy in the form of fast particles very efficiently.

Let us first look at the fragmented situation: As indicated in Figure 1, stellar collisions become important at the density considered. Coalescence leads to more massive stars which rapidly burn out their nuclear fuel and suffer gravitational instability. In other close encounters stars may be torn and will shed much matter into interstellar space. (The same will happen in the collapse events themselves, due to the presence of magnetic fields and rotation.) The resulting situation, with frequent 
local collapses embedded in a dense medium, would certainly lead to quasar-like phenomena (Colgate, 1967; Dyson, 1968). It is very important that we have learned from current pulsar theory (Pacini, 1968; Ostriker and Gunn, 1969; Goldreich and Julian, 1969) how energetic particles may be produced very efficiently. If a neutron

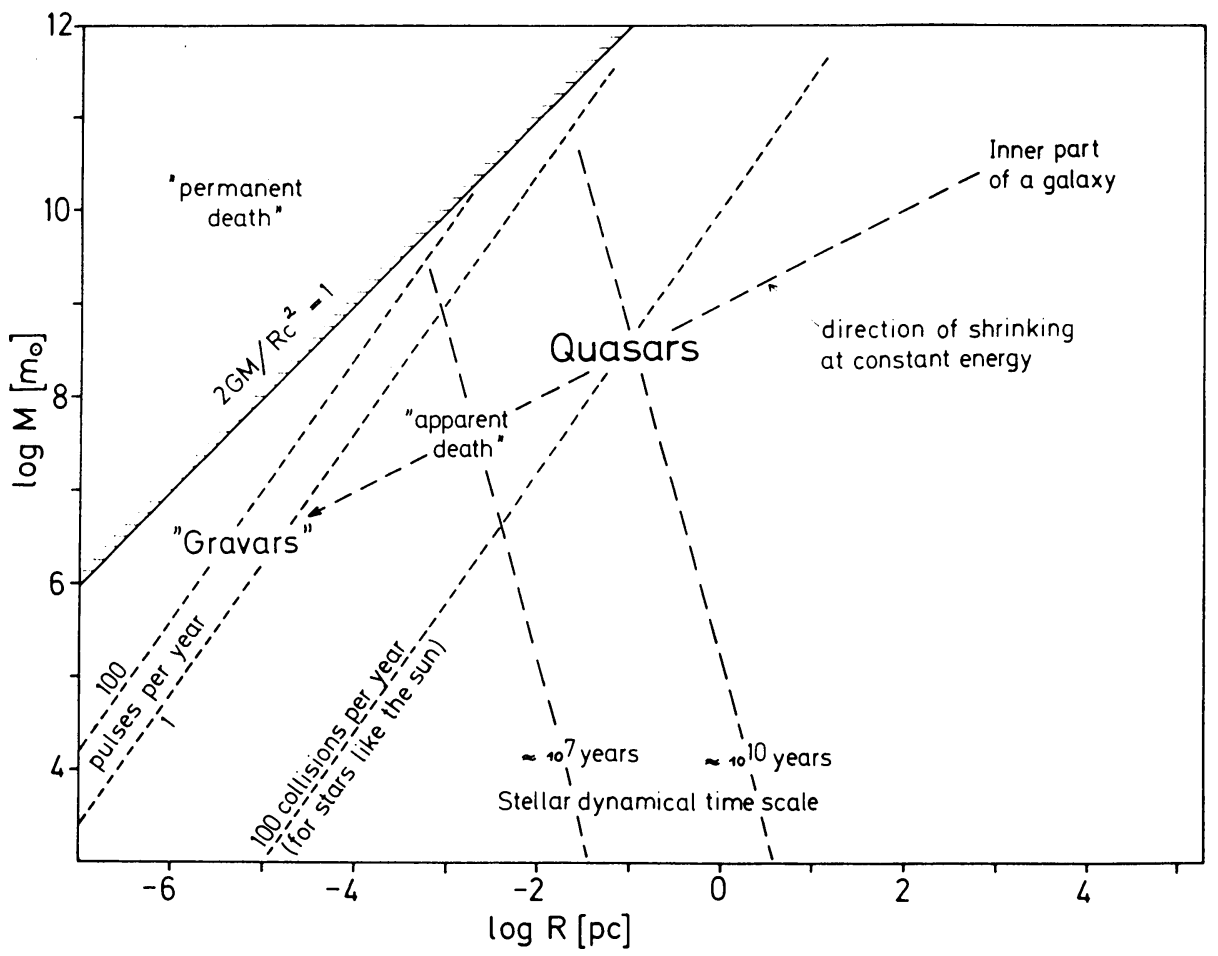

Fig. 1. Mass-radius diagram of clusters of stars (at the right-hand side of the line labelled ' 100 collisions per year') or black holes (at the left-hand side).

star is left after collapse, a considerable fraction of total emission will be concentrated within the first few days. It seems likely, however, that a similar pulse must arise in the formation of a 'rotating black hole', which cannot remain magnetically connected with its neighborhood, and must 'radiate away' the magnetic field.

Many details of final 'Magneto-rotational-gravitational' collapse are still not understood, but it seems more and more likely that there is no severe problem with the conversion of enough gravitational potential energy to fast particles, if rotation and magnetic fields are cooperating with gravitation.

A quasar model of this 'already fragmented' type would not account for periodic activity. Whoever is convinced by observations of such periodicities, must discard this hypothesis or else introduce some coherence of the whole cluster which allows for influence of its overall pulsation or rotation on the rate of local collapses.

If the compact object is still unfragmented, it might work as the now fashionable 
giant pulsar-like machine (Morrison, 1969; Cavaliere et al., 1969; Fowler at the IAU-Symposium No. 46 at Jodrell Bank; Woltjer's review at the present symposium), or the magneto-turbulent 'magnetoid' (Ozernoy and Chertoprud, 1967). If it can remain coherent even through its final states, the remnant will be a single giant black hole (cf. Lynden-Bell, 1969), probably of the Kerr-type (Bardeen, 1970).

However, it seems likely that fragmentation cannot be avoided (cf. Dyson, 1968). In this case, even if part of the catastrophic activity took place during a coherent stage, local instabilities would finally take over and leave us with a (flattened) cluster of collapsed objects, as in the first case.

As long as there are normal stars left, they will collide with each other or with collapsed stars. After some time all stars are collapsed or have been thrown out. As most of the collapsed stars are expected to be heavier than a few suns, they will be (Kerr-type) black holes, and not neutron-stars. Their cross-section is about $10^{10}$ times smaller than before collapse, and hence collisions no longer occur. The only detectable activity might come from the accretion of the dense interstellar medium. This process also brakes the collapsed star and induces a shrinking of the cluster in addition to the stellar dynamical 'evaporation'. But even the latter works now on a time-scale of several $10^{9} \mathrm{yr}$ (neglecting the possibly accelerating modifications mentioned above). Hence, even when most of the gas within the cluster has been swallowed by its members, and the object seems dead, contraction still must go on, and finally the remaining cluster members come so close to each other, that collisions of black holes begin to occur. Fusion or close passage of black holes, however, is certainly the source of strong gravitational radiation. This may appear a rather speculative idea, but a glance at the diagram shows that a quasar remnant will inevitably pass through the critical region, where collisions of black holes become dominant, if fragmentation into stars occurred at any time during the earlier evolution. "For shortness and for fun" the author called such dense clusters of black holes 'gravars' (Kafka, 1969, 1970). During their short life they emit a considerable fraction of their rest energy in pulses of gravitationial radiation which could fit the results reported by Weber $(1969,1970)$, if remnants of a quasar-like stage were situated in the nucleus of our own galaxy. (In the simplest model the collision rates, indicated in the diagram by the lines for 1 and 100 pulses per year, do not depend on the mass of the fragments but only on the total mass $M$.) In a real quasar remnant one might expect some subclustering, such that instead of a single very massive cluster many smaller ones would cross the 'gravar' region, causing strong fluctuations in the gravitational radiative activity.

During the stage of apparent death and in the final 'gravar' phase the quasar emnant must supply its mother-galaxy with a total mass of about $10^{8} M_{\odot}$ in the form of black holes. Immediately after the exhaustion of the quasar activity their ejectionvelocity would be of the order of several thousand $\mathrm{km} \mathrm{s}^{-1}$. In the gravar phase they are thrown out nearly at the velocity of light. When they come to large distances from the cluster, their velocity will on the average have dropped to low values. For some 'tail' of the distribution, however, extreme velocities may survive.

At the very end of such a quasar remnant, this 'sling mechanism' as well as the 
fusion of member-black-holes and the gravitational radiation compete in the destruction of the cluster. Whatever is left must collapse in a single black hole, but very likely this has only a small fraction of the original quasar's mass. All the rest became redistributed in the mother-galaxy in the form of collapsed stars or has been radiated away in pulses of gravitational radiation.

Hence, even if astronomers should find that there are no dark massive objects in the nuclei of galaxies (cf. King's contribution), this would not necessarily mean that there have never been any. If fragmentation occurred, a quasar will not leave much.

\section{References}

Bardeen, J.: 1970, Nature 226, 64.

Cavaliere, A., Pacini, F., and Setti, G.: 1969, Astrophys. Letters 4, 103.

Colgate, S. A.: 1967, Astrophys. J. 150, 163.

Dyson, F. J.: 1968, Astrophys. J. Letters 154, L37.

Ginzburg, V.L. and Ozernoy, L. M.: 1964, Zh.Eksper.Teor. Fiz.47, 1030 (Soviet Phys. JETP 20, 689).

Gold, T.: 1965, in Quasi Stellar Sources and Gravitational Collapse. Proceedings of the first Texas Symposium on Relativistic Astrophysics.

Goldreich, P. and Julian, W. H.: 1969, Astrophys. J. 157, 869.

Von Hoerner, S.: 1968, Bull. Astron. Paris, Ser. 3, 3, 147.

Kafka, P.: 1969, Mitt. Astron. Ges. 27, 134.

Kafka, P.: 1970, Nature 226, 436.

Kardashev, N. S.: 1964, Astron. Zh. 41, 807 (Soviet Astron. 8, 643).

Lynden-Bell, D.: 1969, Nature 223, 690.

Morrison, P.: 1969, Astrophys. J. Letters 157, L73.

Ostriker, J. P. and Gunn, J. E.: 1969, Astrophys. J. 157, 1395.

Ozernoy, L. M.: 1966, Astron. Zh. 43, 300 (Soviet Astron. 10, 241).

Ozernoy, L. M. and Chertoprud, V. E.: 1967, Astron. Zh. 44, 537 (Soviet Astron. 11, 428).

Pacini, F.: 1968, Nature 219, 145.

Piddington, J. H.: 1964, Monthly Notices Roy. Astron. Soc. 128, 345.

Piddington, J. H.: 1967, Monthly Notices Roy. Astron. Soc. 136, 165.

Pikel'ner, S. B.: 1965, Astron. Zh. 42, 3 (Soviet Astron. 9, 1).

Spitzer, L. and Saslaw, W.: 1966, Astrophys. J. 143, 400.

Weber, J.: 1969, Phys. Rev. Letters 22, 1320.

Weber, J.: 1970, Phys. Rev. Letters 24, 276.

\section{Discussion}

Question: Would a black hole, flying through the interstellar medium, be observable?

Kafka: Probably not. Some aspects of this problem have been discussed by Salpeter (E. E. Salpeter: 1964, Astrophys. J. 140, 796), however, especially for high velocities it should be re-examined, if the evidence for evaporation of black holes from the galactic center would grow with a confirmation of Weber's results. 